\title{
A SMALL PLATFORM FOR ASTROPHYSICAL RESEARCH BASED ON THE UPM-SAT 1 SATELLITE OF THE UNIVERSIDAD POLITÉCNICA DE MADRID
}

\author{
A. Sanz-Andrés, J. Meseguer, J.M. Perales and J. Santiago-Prowald
}

IDR/UPM, E.T.S.I. Aeronáuticos, Universidad Politécnica de Madrid, E-28040 Madrid, Spain

\begin{abstract}
UPM-Sat 1 is a small scientific, in-orbit demonstration, educational satellite which has been designed, built, tested, integrated, launched and operated by a team of professors, students, and auxiliary personnel belonging to the Universidad Politécnica de Madrid (UPM). After completion of UPM-Sat 1 Mission a new small satellite, UPM-Sat 2, oriented to low-Earth-orbit scientific mission has been designed. In this paper the different subsystems of UPM-Sat 1 are described and the main characteristics of the second small satellite UPM-Sat 2 are outlined. 02002 COSPAR. Published by Elsevier Science Ltd. All rights reserved.
\end{abstract}

\section{UPM-Sat 1}

On July 7, 1995, this small Spanish satellite was launched in French Guiana. UPM-Sat 1, along with the small French satellite Cerise, of similar geometric and weight characteristics, travelled into space as a secondary payload on flight V75 of an Ariane IV-40 launcher, whose primary client was the military satellite Helios 1A. Since then, UPM-Sat 1 follows a heliosynchronous polar orbit at an altitude of $670 \mathrm{~km}$, orbiting the Earth every 98 minutes, its operational life being seven months (213 days). UPM-Sat 1 payloads were an experimental module to analyse the behaviour of liquids under microgravity conditions, besides other in-orbit demonstration experiments related to attitude control, store-and-forward communications and solar cells technology.

With a total mass of $47 \mathrm{~kg}$, UPM-Sat 1 was designed to comply with the geometry and weight limitations and the rigidity and stress requirements established for the auxiliary or secondary payloads launched by Arianespace (Gonzalez-Folgar et al., 1996a). The satellite is parallelepiped in shape, in order to take full

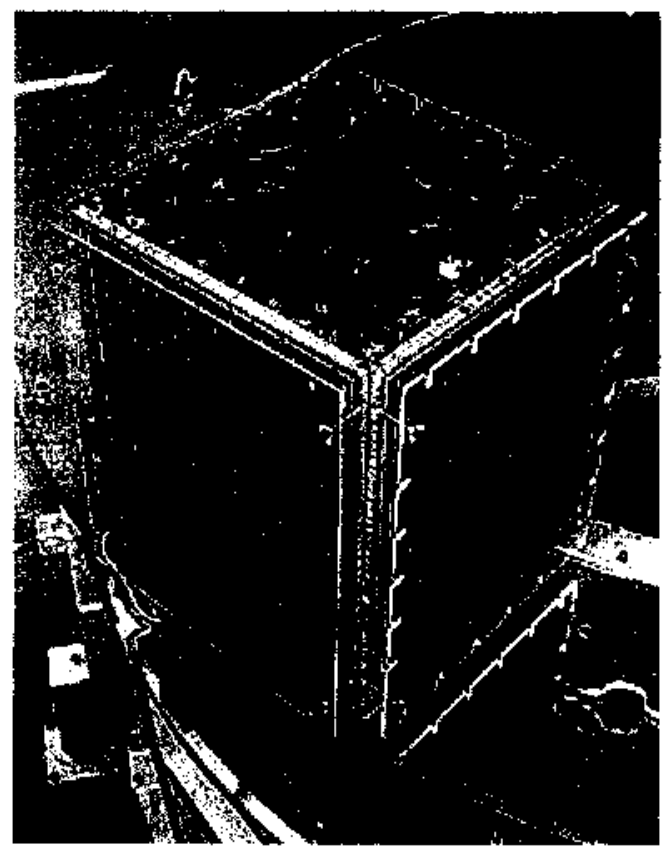

Fig.1. UPM-Sat 1 on the ASAP (Ariane Structure for Auxiliary Payloads). 
advantage of the geometric envelope provided by the launcher. The square base measures $450 \mathrm{~mm}$ each side, with a height of $534 \mathrm{~mm}$, not including the antenna neither the separation system (Figure 1).

The elements conposing the structure of UPM-Sat 1, as sketched in Figure 2, are four trays, separation bars, shear panels, side closure panels and solar panels (Sanz-Andrés et al, 1994, SanzAndres, 1994). The materials used to manufacture the elements of the structure of UPM-Sat 1, were Aluminium-Zinc alloy 7075 T73, Hostaform C9021 (Delrin 07), and stainless steel screws, selected in compliance with the ESA PSS-01-701 standard.

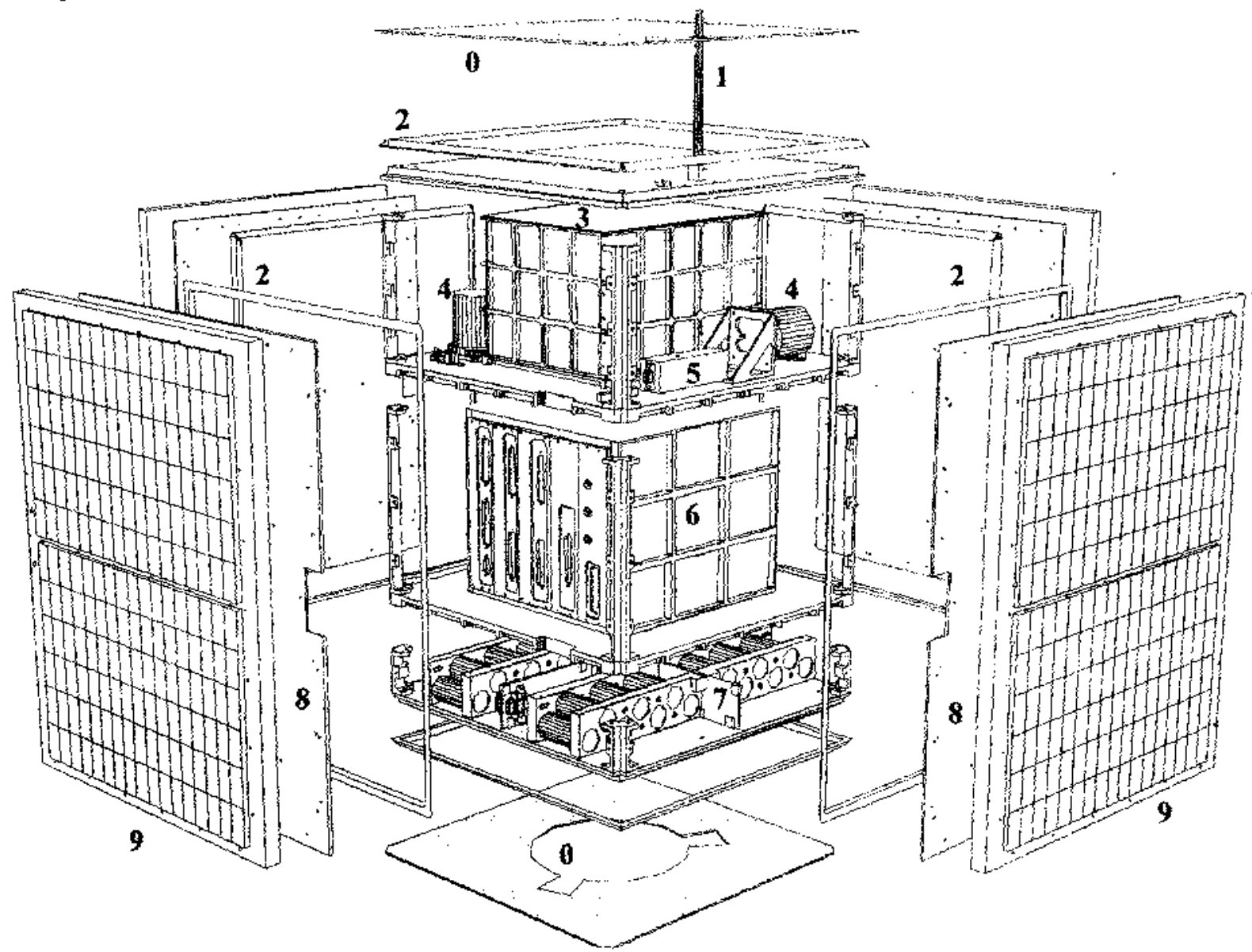

Fig. 2. Exploded view of the UPM-Sat 1:0-multilayer insulation, 1-antenna, 2-magnetic coils, 3-Liquid Bridge Cell, 4- gyroscopes, 5- magnetometers, 6- electronics box, 7- batteries, 8- side panels, 9- solar panel.

The attitude control subsystem is composed of sensors, actuators and control logic. The sensors are two TAM 7 MK2 S triaxial magnetometers, by Ultra Electronics, and three J176 ALM gyroscopes by Sextant Avionique. The actuators are three magnetic coils, two of which are located on the sides of the satellite whereas the third one is located on the lower tray and on the upper tray. The coils were manufactured with 100 spires of enamelled copper wire, produced with Polyester-Polyamide Thetic, class $H$ resins. Each coil is covered with kapton adhesive tape.

To design the thermal protection subsystem the optical properties of the surfaces, those of the aftachments between different elements and components of the satellite, and the use of passive thermal protection elements were all considered. The interior surfaces of the satellite are finished with lowemissivity aluminium, and the inside of the satellite is thermally insulated from the lateral closure panels. In the attachments between trays, separators, panels, etc., low thermal conductivity stainless steel screws were used. Another element of the thermal protection subsystem is the multi-layer thermal insulation 
(MLI) that covers the outside of Trays A and D (see Figures 1 and 2). The MLI contains seven layers of aluminised kapton with fiberglass as a separator. In addition to the above, the satellite revoives in orbit around its longitudinal axis in order to keep the temperature of the solar panels uniform.

Concerning data management, the data acquisition and control module has 54 analog inputs and 3 digital inputs, 4 analog outputs and 19 digital outputs, all related to the measurements and controls of the liquid bridge experiment, attitude control subsystem and power subsystem, as well as temperature measurements and communications with the modem. The intemal sensor module receives 3 temperature signals and 2 analog voltage power signals, and the RS232 interface receives the digital signal for data reception and transmission. The data acquisition, processing and control system uses a $7 \mathrm{MHz} 80 \mathrm{C} 3 \mathrm{I}$ (Matra-Harris) processor, with a MIL STD 883 rating, 256 kbytes SRAM and 64 kbytes EEPROM for programs, watchdog, 64 analog to digital conversion channels of 14-bit resolution, 24 digital outputs, 8 digital inputs and 4 digital to analog conversion channels. Communications with the modem are asynchronous, at $9600 \mathrm{bps}$.

The on-board computer program was designed to perform the following functions: (1) Start/Reset, (2) Ordered shutdown, (3) Performance of experiments and calibrations, (4) Collection of internal status, navigation and time-tagging data, (5) Monitoring of subsystem behaviour (equipment, memory), (6) Compilation of the status/configuration of the satellite, (7) Compilation of the last contacts made, (8) Capacity to establish communication with Earth, (9) Possibility of handling different incidents that could occur, (10) Support for modes of operation (nominal, reduced consumption, lost contact) and (I1) Log of last contacts.

The communications subsystem is basically composed of a quarter wavelength, almost omnidirectional dipole antenna, manufactured in $\mathrm{CuBe}, 180 \mathrm{~mm}$ long and $10 \mathrm{~mm}$ wide, mounted on a piece of Delrin 07 coupled to the upper tray, a modulation/demodulation system made up of a transmitter, a receiver, an antenna changeover switch and a modem, housed in aluminium boxes attachable to the equipment box. The transmission/reception equipment is based on commercial equipment (a $215 \mathrm{~T} 100 \mathrm{~J}$ transmitter and a RCC-100 receiver, both by Aydin). The antenna connector is a Suhner 11 SMA-50-315, which includes a UT 141A cable, items which comply with the MIL-C-39012 standard. For communications, UPM-Sat 1 uses a UHF channel (in the $400 \mathrm{MHz}$ band) with $10 \mathrm{~W}$ of output power at a bandwidth of $200 \mathrm{kHz}$.

The energy management subsystem is composed of three groups of elements, responsible for the generation, storage and management of electrical energy. The energy is generated on the four solar panels located on the sides of the satellite. One part of the mission of UPM-Sat 1 to demonstrate new technologies in orbit- was the testing of two experimental solar panels supplied by ESA/ESTEC (González Folgar et al., 1996b, Ayuso et al., 1996). One solar panel, manufactured by DASA, includes silicon cells and a new aluminium interconnection system; the other is a panel with gallium arsenide cells, produced by FIAR. The other two panels use space proved technology, with silicon cells manufactured by DASA-WEDEL. The panels are rectangular in shape $(425 \times 530 \mathrm{~mm})$, and are each capable of producing $30 \mathrm{~W}$ of power at the beginning of the mission. The total number of cells in the four panels is 696 , and the average power supplied during one orbit is $20 \mathrm{~W}$.

Two batteries were used for energy storage, each consisting of 14 Nickel-Cadmium cells, GATES model GH $6000 \mathrm{~F}$. The two batteries, connected in parallel in order to provide equipment redundancy, supply $6 \mathrm{Ah}$. This subsystem is capable of switching 20 loads with output levels of $5 \mathrm{~V}, \pm 12 \mathrm{~V}, \pm 15 \mathrm{~V}, 24$ $\mathrm{V}$ and $30 \mathrm{~V}$, and also includes control devices for voltage and intensity levels, protection and battery charge control and timing of the latency period after separation.

The separation subsystem, called SSS ASAP (Standard Separation System for Ariane Structure for Auxiliary Payload), was manufactured for Arianespace by Dassault Aviation, and qualified for space use by UPM.

\section{UPM-Sat 2}

The design of the UPM-Sat 2 satellite has been performed assuming a typical mission for this kind of satellites, giving support to a set of experiments as follows: 
- Low earth orbit particle experiment, whose aim is both the study of the particle environment around the Earth by measuring the direction and energy of the particles reaching the onboard sensors and to fine tuning the whole electronics and sensors for more critical missions.

- Antennae technology, focused to the study of the behaviour of new antenna designs (bands, shapes, materials, etc.) in space environment conditions (study of propagation losses, ageing of materials, etc.).

- Low energy electron density experiment. The measurement of the energy distribution of the electrons in a low Earth orbit can help to both collect data which can support the development of theoretical models and to test in orbit the sensors and associated electronics before they can be used in more critical and expensive missions.

- Solar panels technology. As a continuation of the UPM Sat-1 Mission, the aim is to test new developments related to solar panel elements, and to gather data on their behaviour in orbit.

- Laser orbit determination. A set of optical reflector cubes is mounted on the satellite outer surface which allows for the data collection on the satellite position and speed by the laser ranging ground stations. From these data the orbit parameters and their variations can be determined and associated to the atmospheric density variations due to solar activity and to the geo-gravitational field distribution.

The main features of the UPM-Sat 2, shown in Figure 3, are listed in Table 1. Following the UPMSat 1 experience, the electric and electronics systems of the UPM-Sat 2 has been designed according to the following design drivers: 1) modular concept (each module could be redesigned without an impact in the other modules), with careful control of the interface definition (tension and time signal levels, mean and power, etc.) 2) based on previously defined data and power buses, 3) with a high level of autonomy and resilience against failures, and reliability (design based on proved schemes and devices) and 4) taking into account the limitations derived from the mechanical, thermal and radiative space environment requirements.

Table 1. Characteristics of the UPM-Sat 2 satellite.

\begin{tabular}{|c|c|}
\hline Mass & $50 \mathrm{~kg}$ \\
\hline Dimensions & $\begin{array}{l}450 \mathrm{~mm} \times 450 \mathrm{~mm} \times 500 \mathrm{~mm} \text { (antenna not included) plus } 6 \mathrm{~m} \text { boom (when } \\
\text { deployed in orbit) }\end{array}$ \\
\hline Life time & 2 years \\
\hline Structure & $\begin{array}{l}7075 \text { T73 machined; three layers, sheas panels, Aluminium honeycomb solar } \\
\text { panel supports }\end{array}$ \\
\hline Attitude control & $\begin{array}{l}\text { Gravity gradient stabilisation (boom) augmented by magnetic active damping } \\
\text { (magnetorques plus magnetometers) }\end{array}$ \\
\hline Thermal management & Passive (design plus multilayer insulation) \\
\hline Data management & $\begin{array}{l}16 \mathrm{MHz} \text { TMS 320C32 32-bit DSP, 4Mbyte RAM, I Mbyte EEPROM, watch- } \\
\text { dog, } 12 \text { channels 14-bit ADC, two serial channels for communications with } \\
\text { modems, RS-232 channel for debugging. } 3 \mathrm{~W} \text { power consumption, integral } \\
\text { monitoring of voltage and temperature levels, eight channels serial synchronous } \\
\text { SCI (Motorola) bus for internal communications }\end{array}$ \\
\hline Communications & $\begin{array}{l}\text { Two MSK modems at } 9600 \text { baud rate, } 10 \mathrm{~W} \text { onboard transmitter in } 400 \mathrm{MHz} \\
\text { band (downlink) and } 200 \mathrm{MHz} \text { band (uplink), omnidirectional antenna }\end{array}$ \\
\hline Energy management & $\begin{array}{l}\text { Four deployable and two body mounted GaAs solar arrays } 30 \mathrm{~W} \text { each, } 40 \mathrm{~W} \\
\text { orbit averaged power; two batteries } \mathrm{NiCd}, 20 \mathrm{~V} \text { bus bar; energy control card, } \\
\text { load switching control card }\end{array}$ \\
\hline Separation system & SSS ASAP \\
\hline
\end{tabular}

The electric and electronics system is based on a central processor unit (simpler and cheaper than a system based in a distributed configuration) with the appropriate performances, a low-level control statemachine (to take control in case of failure) supported by the following modules: energy generation and control, load switching control, data acquisition, and communications. 


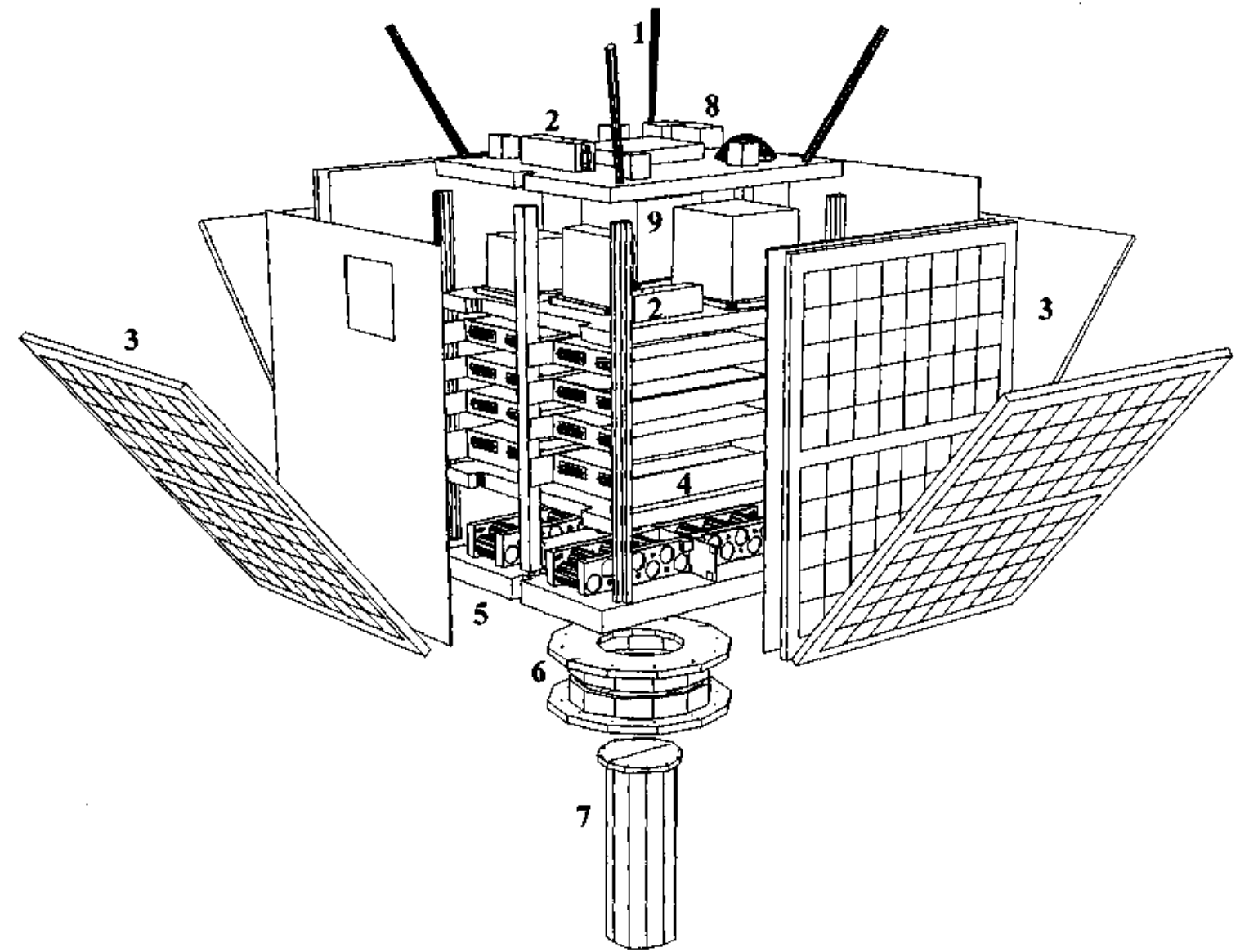

Fig. 3. Exploded view of the UPM-Sat 2: 1- antenna, 2- magnetometers, 3- solar panels (with the magnetic coils wounded around them), 4- electronics box, 5- batteries, 6- separation system, 7- gravity gradient boom, 8- Earth and Sun sensors, 9- payloads (optical cube reflector, K-band experiment antenna and dust sensor).

An eight channels, serial synchronous normalised ( $\mathrm{SCI}$, Motorola) bus is used by the central processor for the communications with both the internal and user modules. This kind of bus is simple, reduces wining and is more reliable than parallel buses.

The modules accept low level control signal coming from the state-machine to allow for failure recovery through ground commands (control processor reset, modules switching, battery charge, etc.)

The functions of the energy generation and control module are the same as for the UPM-Sat 1 . This module works without control of the central processor, independently of the other modules to increase toughness and reliability. An orbit averaged $40 \mathrm{~W}$ power is generated by 6 GaAs solar panels ( 2 body mounted, and 4 deployable) $0.45 \mathrm{~m} \mathrm{x} 0.45 \mathrm{~m}$ each panel. Allowable energy is stored in two independent batteries formed by $14 \mathrm{Ni}$-Cd cells each, leading to a 20 to $25 \mathrm{~V}$ non-regulated bus, with shunt maximum tension protection. It sends housekeeping data (charge and discharge current, temperature and voltage, of batteries, solar panels, bus and module internal systems) to the central processor.

The load switching and control module distributes the energy supplied by the energy generation module to the other modules by using solid state switches controlled by the central processor. The outputs have current limited protection and reseteable fuses. There are two types of outputs: the stabilised ones for the platform modules and the non-regulated (although protected) for the users' modules. This module also sends housekeeping data to the central processor. 
The central processor performs control of the other modules (the platform as well as the users' modules), the acquisition, storage and processing of the data of the other modules, following the instructions of the onboard software. The processor module is based on a TMS 320C32, a 32-bit DSP from Texas Instruments which has been very widely used, with military and extended temperature range versions, which allows for a flexible design and growth, with low power consumption modes and large storage address capacity, 8,16 and 32 bits bus width options, and readily available commercial developing tools and real time operating systems. To decrease the power consumption the processor is driven by a $16 \mathrm{MHz}$ clock. It features watchdog low supply protection, external reset connection (to be used by the control state-machine), and MPSD emulator connection for software development and debugging. This module contains also 4 Mbyte RAM, 1 Mbyte EEPROM (which allows to load new software from ground). It also includes a data acquisition system with 12 channels and a 14-bit ADC, and two independent real time clocks to help the real time software (and the time-tagging of the data and system internal relevant events).

The communication of the central processor with the modems is performed via two serial independently-programmable channels which can reach $115 \mathrm{~K}$ baud rate. A third $\mathrm{RS}-232$ channel is included for connection with a local terminal during debugging sessions. The characteristics of the communications subsystem is summarised in Table 1.

\section{ACKNOWLEDGMENTS.}

These projects have been performed under financial support of the Spanish Comision Interministerial de Ciencia y Tecnología (CICYT).

\section{REFERENCES}

Ayuso, A., E. González-Folgar, L. Hernández, J. Meseguer, J.M. Perales, C. Plaza, C. Ramos, J. Santiago, A. Sanz-Andrés, and C. Terrés, Ground Segment and in Orbit Operation Results of the Satellite of the Universidad Politécnica de Madrid UPM-Sat 1, in International Conference on Small Satellites: Missions and Technology, INTA, Madrid, Spain (1996)

González-Folgar, E., L. Hernández, A. Laverón-Simavilla, J. López-Díez, I. Pérez-Grande, P. Rodríguez de Francisco, J. Santiago, and A. Sanz-Andrés, UPM-Sat 1, the Satellite of the Universidad Politécnica de Madrid: Platform Description, in International Conference on Small Satellites: Missions and Technology, INTA, Madrid, Spain (1996a).

González Folgar, E., Sanz, A., and Larue, J.C., Flight Test of Aluminium Interconnectors for Solar Cells On-Board UPM-Satl, Preparing for the Future, 6, 14 (1996b).

Sanz-Andrés, A., El satélite español científico y de comunicaciones UPM/LB Sat, in ESA SP-363, pp. 325-330, ESA, Paris, France (1994).

Sanz-Andrés, A., J. López-Díez, J.L. Espino, V. Marco-Gómez, and L. Hernández: UPM/LB Sat: Development Status, in Proceedings of the 2nd International Symposium on Small Satellites Systems and Services, pp. 1-15, CNES, Biarritz, France (1994). 